ARTICLE

\title{
Diagnosis and prognosis of myocardial infarction on a plasmonic chip
}

Wei Xu (1) ${ }^{1,2}$, Lin Wang (10 ${ }^{3}$, Ru Zhang (1) 1,2, Xuming Sun (1) 1,2, Lin Huang (1) 1,2, Haiyang Su (1) 1,2, Xunbin Wei ${ }^{1}$, Chia-Chun Chen ${ }^{4}$, Jiatao Lou (10 ${ }^{3 凶}$, Hongjie Dai (i) ${ }^{5}$ \& Kun Qian (1) ${ }^{1,2 \otimes}$

Cardiovascular diseases lead to $31.5 \%$ of deaths globally, and particularly myocardial infarction (MI) results in 7.4 million deaths per year. Diagnosis of $\mathrm{MI}$ and monitoring for prognostic use are critical for clinical management and biomedical research, which require advanced tools with accuracy and speed. Herein, we developed a plasmonic gold nano-island ( $p$ Gold) chip assay for diagnosis and monitoring of MI. On-chip microarray analysis of serum biomarkers (e.g., cardiac troponin I) afforded up to 130-fold enhancement of near-infrared fluorescence for ultra-sensitive and quantitative detection within controlled periods, using 10 $\mu \mathrm{L}$ of serum only. The pGold chip assay achieved MI diagnostic sensitivity of $100 \%$ and specificity of $95.54 \%$, superior to the standard chemiluminescence immunoassay in cardiovascular clinics. Further, we monitored biomarker concentrations regarding percutaneous coronary intervention for prognostic purpose. Our work demonstrated a designed approach using plasmonic materials for enhanced diagnosis and monitoring for prognostic use towards point-of-care testing.

\footnotetext{
${ }^{1}$ School of Biomedical Engineering and Med-X Research Institute, Shanghai Jiao Tong University, Shanghai 200030, China. ${ }^{2}$ State Key Laboratory for Oncogenes and Related Genes, Division of Cardiology, Renji Hospital, School of Medicine, Shanghai Jiao Tong University, 160 Pujian Road, Shanghai 200127, China. ${ }^{3}$ Department of Laboratory Medicine, Shanghai Chest Hospital, Shanghai Jiao Tong University, Shanghai 200030, China. ${ }^{4}$ Department of Chemistry, National Taiwan Normal University, Taipei 11677, Taiwan. ${ }^{5}$ Department of Chemistry, Stanford University, Stanford, CA 94305, USA.

凶email: loujiatao@shchest.org; k.qian@sjtu.edu.cn
} 
W hile cardiovascular diseases (CVDs) threaten human health with the lifetime risk exceeding $30 \%$, myocardial infarction (MI) is the leading cause of death for CVDs nowadays ${ }^{1,2}$. Notably, the influence of MI is becoming more severe in recent decades with an annual growth rate of over $3.6 \%{ }^{3,4}$, due to changes in human behavior and lifestyle, particularly in developing countries ${ }^{5,6}$. Considering the substantial progress in the treatment of MI, current management of MI is focused on diagnostic and prognostic approaches, which can reduce the medical costs and raise the survival ratio as well as life quality of patients ${ }^{7,8}$. Therefore, an advanced analytical tool would be of key significance toward efficient management of MI.

Blood test serves as the major clinical method for monitoring of diseases including but not limited to $\mathrm{MI}^{9,10}$. Blood tests are convenient, noninvasive, and accurate, superior to the conventional methods such as the electrocardiogram (ECG) and physical examination $(\mathrm{PE})^{11,12}$. Nevertheless, the effectiveness of blood tests in clinics relies on designed materials and device, considering the following aspects: (I) high sensitivity and specificity for precision diagnostics; (II) low sample volume for point-ofcare testing (POCT); (III) disease-specific performance with tailored analytical features (e.g., speed and selection of biomarkers); and (IV) multi-functions in clinical application, such as monitoring for prognostic use ${ }^{13,14}$. To date, the present blood tests are still far from ideal for monitoring of MI and call for new platforms addressing the above aspects.

Plasmonic materials are usually noble metals and afford unique optical properties for diverse biomedical use ${ }^{15-17}$. Particularly, nearinfrared fluorescence enhanced (NIR-FE, the wavelength of 650-1700 nm) detection promises the state-of-the-art in vivo imaging and in vitro diagnosis, through a series of pre-selected materials interfaces ${ }^{18-20}$. For example, given characteristic gold nanoislands and immobilized immunoassays on the surface, plasmonic gold nano-island (pGold) chips afford NIR-FE detection of target molecules by high-performance microarrays ${ }^{21}$. NIR-FE detection has engaged in diagnostics including cancer $^{22}$, diabetes ${ }^{23,24}$, hypertensive heart disease ${ }^{25}$, and infectious diseases et ${ }^{26}$. To date, the application of NIR-FE in MI has not been established and a further obstacle is monitoring for prognostic use in clinics, both of which should be tackled due to the urgent needs for better MI management and multi-use in clinical laboratory and POCT.

Herein, a plasmonic gold nano-island chip based assay was developed for the diagnosis and monitoring of MI. On-chip microarray analysis of serum biomarkers (e.g., cardiac troponin I) afforded up to 130 -fold enhancement of near-infrared fluorescence for ultrasensitive and quantitative detection within controlled periods (down to $30 \mathrm{~min}$ ), using $10 \mu \mathrm{L}$ of serum only. We achieved MI diagnostic sensitivity of $100 \%$ and specificity of 95.54\% with area under the curve (AUC) of 0.976 (95\% confidence interval (CI): $0.950-1.000, p=2.85 \times 10^{-11}$ ), superior to the standard chemiluminescence immunoassay in cardiovascular clinics. We performed post-operative monitoring of percutaneous coronary intervention (PCI, the universal treatment procedure for MI). Our work demonstrated a designed approach using plasmonic materials for enhanced diagnosis and monitoring for prognostic use toward point-of-care testing.

\section{Results}

Construction and characterization of pGold chip assay. The sandwich immunoassay was constructed toward selected biomarkers on pGold chips (Fig. 1a). pGold chips (obtained from Nirmidas Biotech Inc.) afforded unique extinction spectra (Fig. 1b) and discontinuous gold nano-islands on the surface for NIR-FE detection (Fig. 1c). As shown in the high-resolution top-view SEM image inset of Fig. 1c, there are gold nano-islands with size of
$80-200 \mathrm{~nm}$ and gaps of $10-30 \mathrm{~nm}$. The wavelength of the plasmon band was $\sim 610 \mathrm{~nm}$ for plasmonic gold nano-island chip and $\sim 570$ $\mathrm{nm}$ for sputter gold (sGold) chip ${ }^{21,27}$. We printed the triplicate microarrays of antibodies on the pGold chip (digital images in Fig. 1d, e) and assembled the device capable of 16 samples detection in parallel (Supplementary Fig. 1) for high throughput application.

The pGold chips had a surface layer of gold with a thickness of $\sim 80 \mathrm{~nm}$ and microarray printing introduced the additional layer of antibodies, according to the cross-section SEM in Fig. 1d, e. The distribution of antibodies aggregates on the surface was revealed by the top-view SEM in Fig. If, consistent with the crosssection SEM. Therefore, using the as-printed pGold chips to quantitate serum biomarkers, immunoassays can be conducted within the optimized proximity to plasmonic surface ( hundreds of nanometers $)^{21,22}$ for NIR-FE detection.

NIR-FE detection of serum biomarkers on-chip. The pGold chips enhanced NIR fluorescence up to 130-fold in the detection of cardiac troponin I (cTnI), compared with the glass chips according to fluorescence mapping results (Fig. 2a, Supplementary Tables 1,2). The sGold chips with continuous gold film afforded reduced fluorescence due to the surface quenching effect ${ }^{28}$. For glass chips, the silica glass afforded no fluorescence enhancement due to nonplasmonic resonances of bulk silicate $e^{29,30}$ and only weak signals of fluorophore IRDye800 with a quantum yield of $0.05-0.1 \%^{21,31} \mathrm{can}$ be observed, for $0.3 \mathrm{ng} \mathrm{mL}^{-1}$ cTnI with average fluorescence intensity of 43.84 after background noise subtraction. For sGold chips, the continuous gold film resulted in a decrease of quantum yield and quenching of fluorescence ${ }^{32,33}$, for $0.3 \mathrm{ng} \mathrm{mL}^{-1} \mathrm{cTnI}$ with average fluorescence intensity of $\sim 0$ after background noise subtraction. For pGold chips, the discontinuous gold nanoislands produced surface plasmon resonance under $\operatorname{laser}^{22,25}$ and the enhanced near-infrared fluorescence can be obtained, for $0.3 \mathrm{ng}$ $\mathrm{mL}^{-1} \mathrm{cTnI}$ with average fluorescence intensity of 5727.22 after background noise subtraction. Consequently, near-infrared fluorescence enhanced detection can be obtained on pGold chips, compared with the glass chips $(p<0.0001)$ and sGold chips $(p<0.0001)$, respectively. Therefore, only the gold nano-island platform (pGold chips) yielded the expected results.

Calibration curves were recorded for $\mathrm{cTnI}$ in a dynamic range of $0.01-1.20 \mathrm{ng} \mathrm{mL}^{-1}$ on the pGold chips (Fig. $2 \mathrm{~b}$, superior to glass and sGold chips), with limit-of-detection (LOD) and limitof-quantification (LOQ) of 0.0100 and $0.0157 \mathrm{ng} \mathrm{mL}^{-1}$, respectively (Table 1 ). The results demonstrated the improved LOD and LOQ of pGold chip in detecting cTnI $(p<0.05)$, compared with the chemiluminescence immunoassay (CIA, the current gold standard in serum biomarkers based MI diagnosis) in Fig. 2c, Table 1, and Supplementary Table 1. Specifically, we calculated the LOD of pGold chip assay using the following Eq. (1):

$$
\begin{aligned}
{[\mathrm{LOD}]=} & {[\text { mean blank value }]+3 \times[\text { standard deviation }(\mathrm{SD})} \\
& \text { of the blank value }]
\end{aligned}
$$

which had been reported in previous literatures ${ }^{22,23,26}$

For comparison, the LOD in high-sensitivity cTnI assays was calculated using the following Eqs. (2) and (3):

$$
\begin{aligned}
{[\mathrm{LOD}]=} & {[\text { limit-of-blank }(\mathrm{LOB})]+1.645 \times[\mathrm{SD} \text { of the lowest }} \\
& \text { measurable cTnI value } \left.\left(0.01 \mathrm{ng} \mathrm{mL}^{-1} \text { in our work }\right)\right]
\end{aligned}
$$

$$
[\mathrm{LOB}]=[\text { mean blank value }]+1.645 \times[\mathrm{SD} \text { of the blank value }]
$$

which was also reported in previous literatures ${ }^{34-36}$. 
a

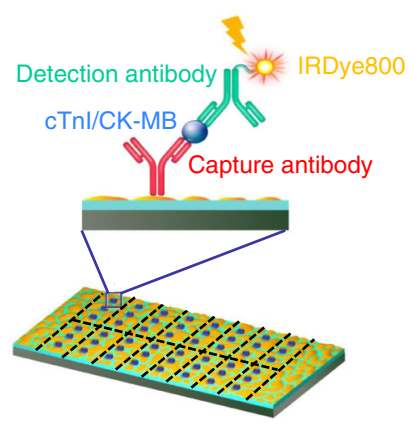

d

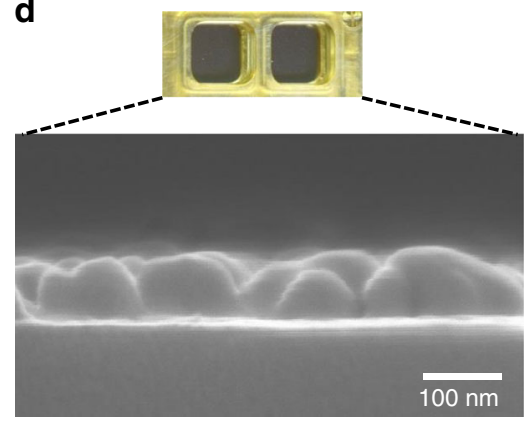

b

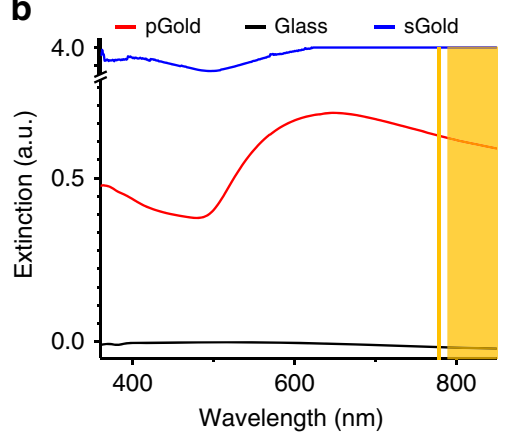

e

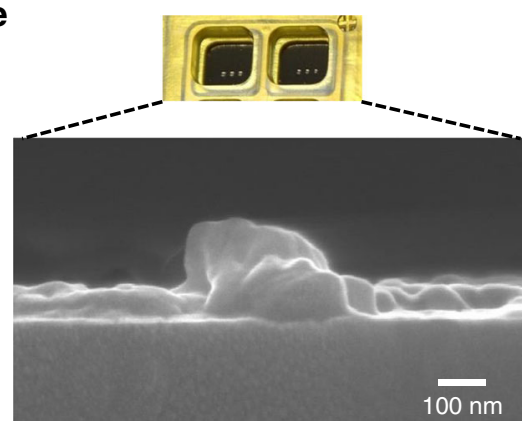

C

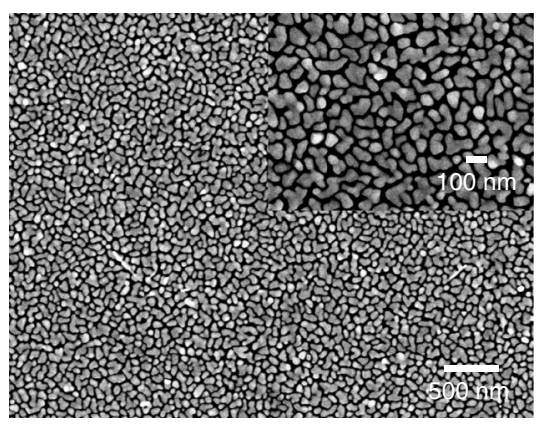

f

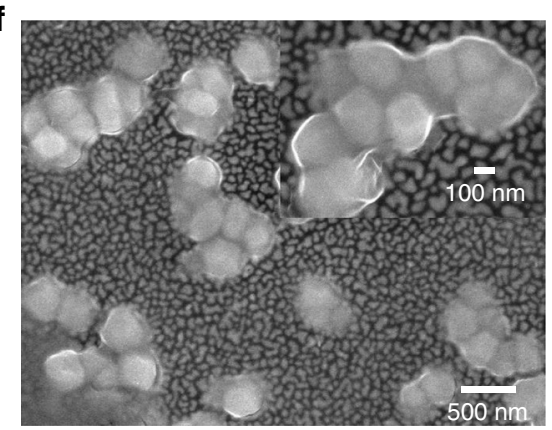

Fig. 1 Construction and characterization of antibody-printed pGold chips. a Schematic illustration of antigen (cTnl/CK-MB) detection on pGold chip using a sandwich assay. b Extinction spectra of pGold chip (red line), glass (black line), and sGold chip (blue line) overlaid with the excitation and emission (yellow shaded area) regions of IR800 dye. c Top-view SEM of pGold chip ( $n \geq 3$ randomly selected). d Digital image of two bulk reaction wells and sideview SEM of gold islands on pGold chip. e Digital image of two antibody-printed reaction wells and side-view SEM of capture antibodies on pGold chip. f Top-view SEM of capture antibodies on pGold chip ( $n \geq 3$ randomly selected). Insets of (c) and (f) were the zoomed SEM images. Source data are provided as a Source Data file.

Notably, the LOD of pGold chip assay was calculated to be $0.0030 \mathrm{ng} \mathrm{mL}^{-1}\left(3 \mathrm{ng} \mathrm{L}^{-1}\right)$ based on the same equation as highsensitivity cTnI assays (Eq. (2)), comparable to the LOD of current high-sensitivity cTnI assays $\left(1-5 \mathrm{ng} \mathrm{L}^{-1}\right)$. Moreover, the cutoffs of current high-sensitivity cTnI assays are set to be 14-52 ng $\mathrm{L}^{-1}$ (refs. ${ }^{37-39}$ ), even for $0 / 1 \mathrm{~h}$-algorithm or $0 / 2 \mathrm{~h}$-algorithm, according to the guidelines of commercial diagnostic kits and literature reports, to avoid false positives and achieve high diagnostic specificity (>95\%). Therefore, the LOD of pGold chip assay $\left(3 \mathrm{ng} \mathrm{L}^{-1}\right)$ is comparable to the LOD of current highsensitivity cTnI assays $\left(1-5 \mathrm{ng} \mathrm{L}^{-1}\right)$ and sufficient for clinical use considering the recommended cutoffs (14-52 $\left.\mathrm{ng} \mathrm{L}^{-1}\right)$.

Sensitivity is fundamentally important for the detection of serum biomarkers for efficient diagnosis of MI, due to the low concentration of biomarkers in the early stages of the disease. Similar results were obtained through NIR-FE detection of creatine kinase isoenzyme $\mathrm{MB}$ (CK-MB, Fig. 2d) on the pGold chips, showing advantages of pGold as a platform technology (Fig. 2e, f, Table 1, Supplementary Table 3). Notably, NIR-FE of cTnI and CK-MB on pGold chips yielded coefficient variation < $15 \%$ (Supplementary Tables 4,5 ) in the calibration curve for low concentrations $\left(0.01-1.20 \mathrm{ng} \mathrm{mL}^{-1}\right.$ for cTnI and $0.25-64.00 \mathrm{ng}$ $\mathrm{mL}^{-1}$ for CK-MB, Supplementary Tables 1,3), validating the reproducibility and accuracy of our assay for clinical use.

Diagnosis of MI patients by serum biomarkers. We detected 10 $\mu \mathrm{L}$ of serum samples from $112 \mathrm{MI}$ patients $(75$ males and 37 females, with median age of 64.39 , who were diagnosed with MI and without other major diseases on the basis of symptoms of myocardial ischemia, elevated cardiac biomarker, the electrocardiogram (ECG), and angiography) and 112 healthy controls (81 males and 31 females with median age of 61.66 , who were diagnosed without MI and other major diseases) by the pGold chip assay for diagnosis of MI (Supplementary Table 6). Patients/ controls known to have other medical conditions (such as active bleeding) were excluded. Both age and gender were matched with $p>0.05$ (Supplementary Table 6, according to Student's $t$-test and Fisher's exact test), between the MI patients and healthy controls.

According to the fluorescence intensity from patients and controls (NIR-FE detection in Fig. 3a, CIA results in Supplementary Fig. 2a), the corresponding receiver operating characteristic (ROC) curves of cTnI were plotted for MI diagnosis (Fig. 3b). The pGold chip assay and CIA afforded AUC of 0.976 (95\% CI: 0.950-1.000, $p$ $=2.85 \times 10^{-11}$ ) and 0.662 (95\% CI: $0.590-0.734, p=0.000023$ ), respectively, in Table 2 . The serum samples were diluted to the same volume $(90 \mu \mathrm{L})$ by fetal bovine serum (FBS) for both plasmonic sensing platform and conventional approach (CIA), to test these two different methods under the same sample conditions. We used the CIA on the UniCel DxI 800 platform (Beckman Coulter), which is a reported high-sensitivity cTnI assay in clinic use according to current guidelines ${ }^{40}$ and requires $200 \mu \mathrm{L}$ of serum for the optimal standard of care use. The decrease of sample volume ( $10 \mu \mathrm{L}$ of serum in this work) resulted in the poor performance of the test. We used the same sample volume ( $10 \mu \mathrm{L}$ of serum) for both CIA and pGold chip assays, to make a valid comparison.

Superior to CIA with diagnostic sensitivity of $59.82 \%$ and specificity of $70.54 \%$, the pGold chip assay afforded markedly improved diagnostic sensitivity of $92.86 \%$ and specificity of $97.32 \%$, based on the recommended cutoff concentration by CIA $\left(0.04 \mathrm{ng} \mathrm{mL}^{-1} \text { for cTnI, Tables } 1,2\right)^{41-43}$, due to the enhanced LOD and LOQ. Notably, we achieved further enhanced diagnostic sensitivity of $100 \%$ and specificity of $95.54 \%$, based on the lowered cutoff concentration by pGold $\left(0.03 \mathrm{ng} \mathrm{mL}^{-1}\right.$ for cTnI, Fig. 3, Tables 1,2), which is sufficient to be of clinical use and comparable to Food and Drug Administration (FDA) approved 
a

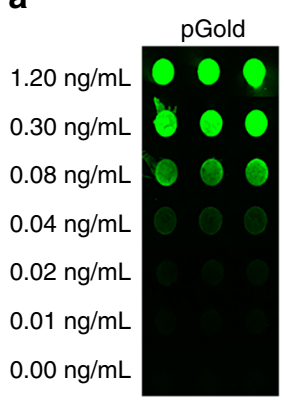

d
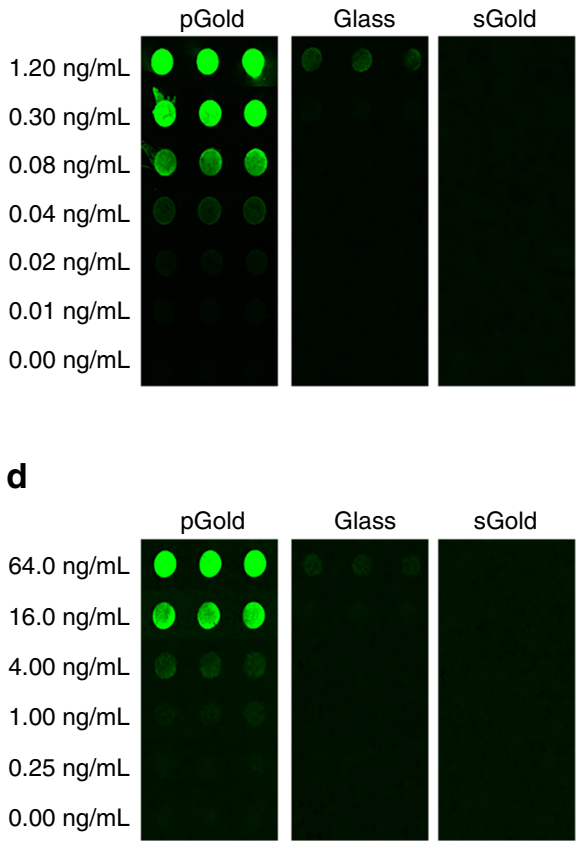

b

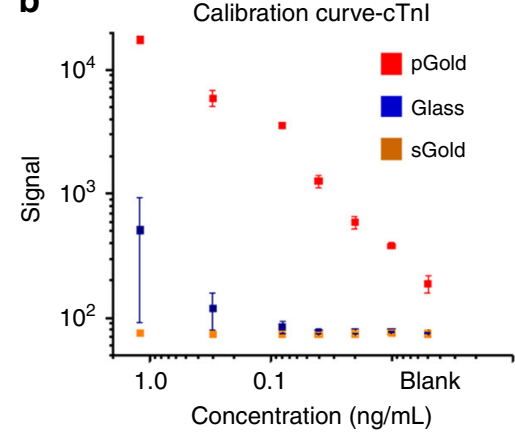

e

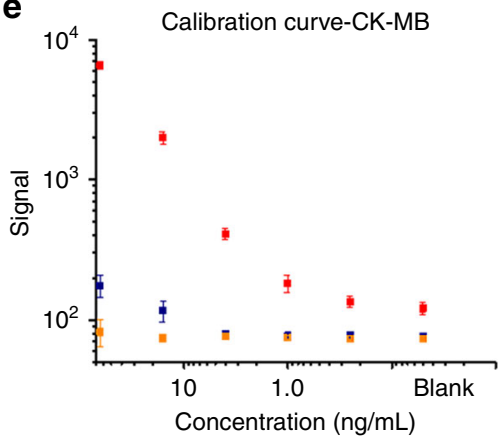

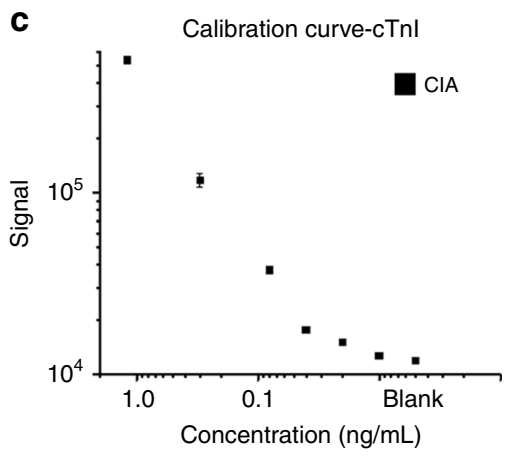

f

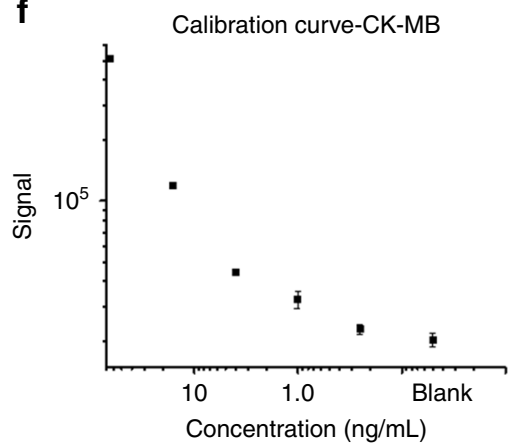

Fig. 2 Near-infrared fluorescence enhanced microarray detection of biomarkers. a Fluorescence mapping results by IRDye800 labeled detection of cTnl (0-1.2 ng mL ${ }^{-1}$ ) on pGold (left), glass (middle), and sGold (right) chips. b Calibration curves comparing detection limits and dynamic ranges for cTnl on pGold (red), glass (blue), and sGold (yellow) chips. We performed three independent experiments on pGold, glass, and sGold chips to calculate the standard deviation (s.d.) as error bars and data are shown as the mean \pm s.d. $(n=3)$. c Calibration curve of cTnl quantification by CIA. We performed three independent $\mathrm{CIA}$ experiments and data are shown as the mean \pm s.d. $(n=3)$. Similarly, d fluorescence mapping results, e calibration curves comparing detection limits and dynamic ranges by different chips, and $\mathbf{f}$ calibration curve by $\mathrm{CIA}$ were obtained for CK-MB. We performed three independent

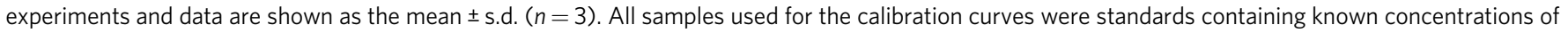
biomarkers provided by the vendor (Tellgen). Source data are provided as a Source Data file.

Table 1 LODs, LOQs, and cutoffs using pGold chip and CIA.

\section{cTnl (ng mL ${ }^{-1}$ )}

CIA-LODa

CIA-LOQb

CIA-cutoffs

pGold-LODa

pGold-LOQb

pGold-cutoffs

0.0152

0.0208

0.04

0.0100

0.0157

0.03

aLODs were calculated by applying the mean blank value plus 3 times of s.d. to the fitting line of calibration curves.

bLOQs were calculated by applying the mean blank value plus 10 times of s.d. to the fitting line of calibration curves.

assays for MI (with sensitivity $>95 \%$ and AUC $>0.96)^{44,45}$. We have also added the results using $200 \mu \mathrm{L}$ of serum for cTnI detection by CIA (Supplementary Fig. 3, with diagnostic sensitivity of $100 \%$ and specificity of $96.43 \%$ based on the lowered cutoff concentration of $0.03 \mathrm{ng} \mathrm{mL}^{-1}$ for $\mathrm{cTnI}$ ), as the supporting data for the direct comparison. Also, we used Abbott Architect kit and i2000SR platform (consuming $200 \mu \mathrm{L}$ of serum), which is a reported high-sensitivity cardiac troponin I (hs-cTnI) assay according to the current guidelines ${ }^{37,40,46}$. The hs-cTnI assay (Architect) afforded the diagnostic sensitivity of $100 \%$, specificity of $94.64 \%$, and AUC of 0.994 (95\% CI: 0.987-1.000) based on the cutoff concentration of $0.03 \mathrm{ng} \mathrm{mL}^{-1}$ (CIA* in Fig. 3b, Supplementary Fig. 4). Therefore, the pGold chip platform achieved high-sensitivity cTnI detection using $10 \mu \mathrm{L}$ of serum only, compared with CIA using $200 \mu \mathrm{L}$ of serum. And the diagnostic performances of two assays were comparable. The success can be due to the highly sensitive troponin testing based on the plasmonic platform with NIR-FE detection, which is highly sensitive and accurate even at very low concentrations.

In parallel, the diagnostic performance of CK-MB (Fig. 3c, Supplementary Fig. 2b) was studied and the corresponding ROC curves were recorded (Fig. 3d). Limited AUC of 0.821 (95\% CI: $0.786-0.886, p=0.000338$, sensitivity of $86.61 \%$ and specificity of $66.07 \%)$ was obtained for CK-MB using the pGold chip assay superior to CIA with AUC of 0.614 (95\% CI: $0.537-0.692, p=$ 0.000493 , sensitivity of $24.11 \%$ and specificity of $98.21 \%$ ), based on the recommended cutoff concentration by CIA $\left(4 \mathrm{ng} \mathrm{mL}^{-1}\right.$ for CK$\mathrm{MB}$, Tables 1,2). Still unsatisfactory diagnostic sensitivity of $86.61 \%$ and specificity of $66.07 \%$ were obtained using the pGold chip assay, based on the lowered cutoff concentration by pGold $\left(3.13 \mathrm{ng} \mathrm{mL}^{-1}\right.$ for CK-MB, Fig. 3, Tables 1, 2), which was consistent with previous literatures ${ }^{47,48}$ and confirmed the key role of cTnI over CK-MB in the diagnosis of MI. Therefore, it was demonstrated that NIR-FE detection of selected biomarkers (cTnI) by the pGold chip assay achieved high diagnostic capability (AUC of 0.976) with a low sample volume of $10 \mu \mathrm{L}$ of serum only, in screening of MI toward potential POCT, which is consistent with previous literature and confirms the key role of cTnI in the diagnosis of MI.

The high specificity of $>95 \%$ referred to the diagnostic specificity, which can be attributed to both the rational selection of biomarker (cTnI for MI patients) and sensitive detection of biomarker (LOD of $0.01 \mathrm{ng} \mathrm{mL}^{-1}$ ). For selection of biomarker, the cTnI was a better biomarker for MI patients due to the characteristic release of cTnI 

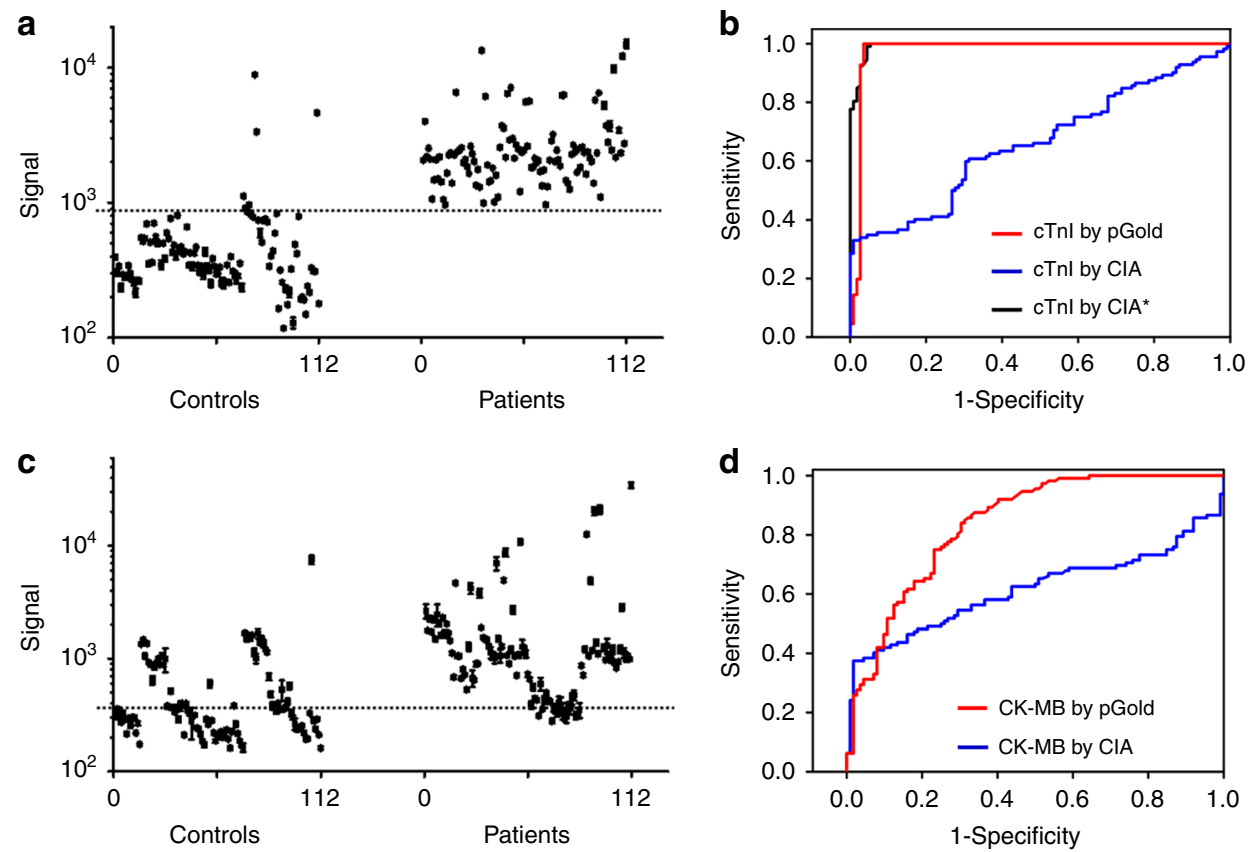

Fig. 3 Serum tests for diagnosis of MI using cTnI and CK-MB. a Signal quantification on pGold chips for the detection of 112 MI patients and 112 controls using cTnl. All experiments were conducted with $n=3$; mean \pm s.d. $\mathbf{b}$ ROC curves for diagnosis by cTnl. The red and blue lines represented $p G o l d$ chips and $\mathrm{CIA}$, respectively. The black line $\left(\mathrm{CIA}^{\star}\right)$ represented the $\mathrm{CIA}$ based on the Abbott Architect assay. $\mathbf{c}$ Signal quantification on pGold chips for the detection of $\mathrm{MI}$ patients and controls using CK-MB. All experiments were conducted with $n=3$; mean \pm s.d. $\mathbf{d}$ ROC curves for diagnosis by CK-MB. The red and blue lines represented pGold chips and $\mathrm{CIA}$, respectively. The dashed lines were the intensity cutoffs corresponding to serum concentrations of $0.03 \mathrm{ng} \mathrm{mL}^{-1}$ for $\mathrm{cTnl}$ and $3.13 \mathrm{ng} \mathrm{mL}^{-1}$ for CK-MB. Source data are provided as a Source Data file.

\begin{tabular}{|c|c|c|}
\hline & CIA $^{\mathbf{a}}$ & pGoldb \\
\hline Sensitivity-cTnl & $59.82 \%$ & $100 \%$ \\
\hline Specificity-cTnl & $70.54 \%$ & $95.54 \%$ \\
\hline$A \cup C-c T n l^{C}$ & 0.662 & 0.976 \\
\hline $95 \% \mathrm{Cl}^{\mathrm{d}}$ & $0.590-0.734$ & $0.950-1.000$ \\
\hline$p$ value-cTnle & 0.000023 & $2.85 \times \mathrm{E}^{-11}$ \\
\hline Sensitivity-CK-MB & $24.11 \%$ & $86.61 \%$ \\
\hline Specificity-CK-MB & $98.21 \%$ & $66.07 \%$ \\
\hline$A \cup C-C K-M B C$ & 0.614 & 0.821 \\
\hline $95 \% \mathrm{Cl}^{\mathrm{d}}$ & $0.537-0.692$ & $0.786-0.886$ \\
\hline$p$ value-CK-MBe & 0.000493 & 0.000338 \\
\hline
\end{tabular}

aSensitivity and specificity by $\mathrm{ClA}$ were obtained at the cutoffs of $0.04 \mathrm{ng} \mathrm{mL}-1$ for $\mathrm{cTnl}$ and 4 ng $\mathrm{mL}^{-1}$ for CK-MB

bSensitivity and specificity by pGold chip were obtained at the lowered cutoffs of $0.03 \mathrm{ng} \mathrm{mL}^{-1}$ for $\mathrm{CTnl}$ and $3.13 \mathrm{ng} \mathrm{mL}^{-1}$ for CK-MB.

cAUCs were obtained using the SPSS software (version 19.0, SPSS Inc., Chicago).

${ }^{\mathrm{d}} \mathrm{Cl}$ was calculated based on the Clopper-Pearson method.

$\mathrm{e}_{p}$-Value was calculated based on the two-sided Student's t-test.

from myocardial cell to blood, compared with other biomarkers (e.g., CK-MB), according to literature reports ${ }^{47,48}$. For detection of biomarker, sensitive detection of cTnI with LOD of $0.01 \mathrm{ng} \mathrm{mL}^{-1}$ may lead to the clear differentiation between early MI patients with higher cTnI concentrations and healthy controls with lower cTnI concentrations ${ }^{49,50}$, superior to other platforms (e.g., glass chips and sGold chips) with lower detection sensitivity. Therefore, we identified healthy controls with lower cTnI concentrations at high confidence (specificity of $>95 \%$ ) by the rational selection and sensitive detection of the biomarker.

Speed and multiplexity of pGold chip assay. Considering emergencies in real case calling for fast diagnosis of MI, the speed of the pGold chip assay was investigated. NIR-FE detection of cTnI was performed at low concentrations of $0.01-0.08 \mathrm{ng} \mathrm{mL}^{-1}$ (Fig. 4a), with controlled overall immuno-reaction time of 150/ $60 / 30 \mathrm{~min}$ on-chip. The corresponding calibration curves displayed reduced NIR fluorescence intensities by $15-50 \%$ (Fig. $4 \mathrm{~b}$ ). Notably, the LOD and LOQ reached 0.0152 and $0.0331 \mathrm{ng} \mathrm{mL}^{-1}$, based on $30 \mathrm{~min}$ immuno-reaction on chip (Supplementary Table 7). It has been anticipated that with further instrumentation and automation, our pGold chip assay can engage the requirement for the diagnosis of $\mathrm{MI}$ in a much shorter time for emergencies.

Also, multiplex detection of biomarkers was conducted on the pGold chips. Capture antibodies for cTnI and CK-MB were applied in a $3 \times 2$ spot matrix format with each row containing one type of capture antibodies. For specificity tests, we implemented one detection antibody for biomarkers with different concentrations (0.3-1.2 $\mathrm{ng} \mathrm{mL}^{-1}$ for cTnI, $4.0-64.0 \mathrm{ng} \mathrm{mL}^{-1}$ for CK-MB) and observed bright fluorescent spots in the target row only (Fig. 4c, Supplementary Fig. 5a). Averaged fluorescence intensities of spots in each row were summarized in Fig. 4d, Supplementary Fig. 5b. These data demonstrated the capability of pGold chip assay to carry out multiplex biomarker analysis.

Post-operative monitoring of MI patients after PCI. Further, the serum concentrations of cTnI were monitored to check the health conditions of MI patients after PCI (Fig. 5). A cohort of 25 MI patients (19 males and 6 females) were analyzed with a median age of 68 (41-92). For past medical history, 5 patients had high blood pressure, 14 patients had no related diseases, and 6 patients had MI within recent 3 years (Supplementary Table 8). Three blood samples per patient were collected and monitored including one blood sample before PCI and two blood samples within 10 days (one on day 5 and the other on day 10) after PCI. Specifically, it was found that serum concentrations of cTnI 
a

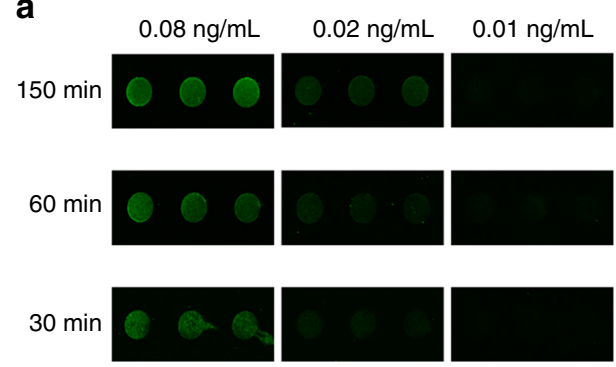

C

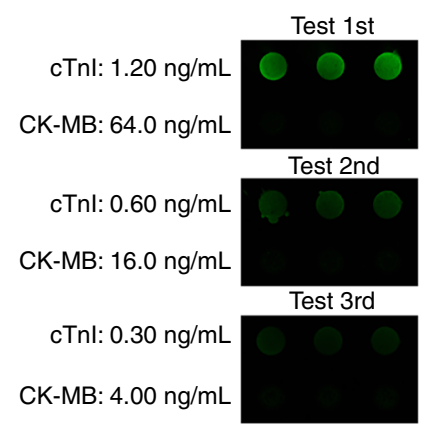

b

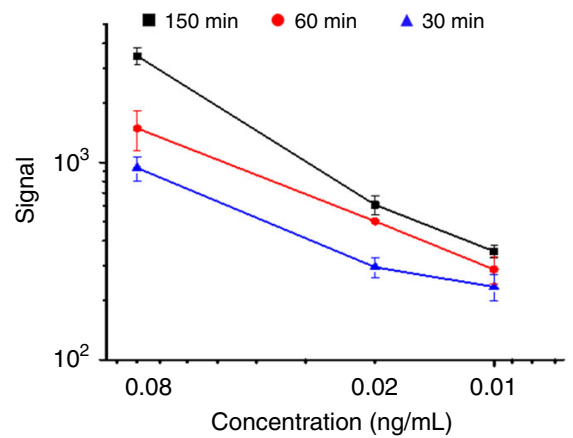

d

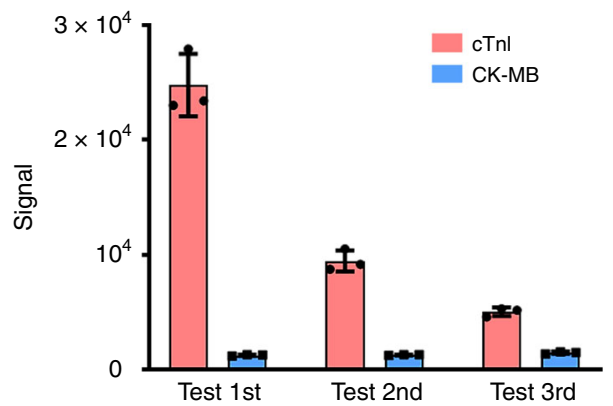

Fig. 4 Control of reaction time and cross reactivity tests. a Fluorescence mapping results and $\mathbf{b}$ corresponding calibration curves with the reaction time of 150,60 , and $30 \mathrm{~min}$ for $\mathrm{cTnl}\left(0.01-0.08 \mathrm{ng} \mathrm{mL}^{-1}\right)$. All experiments were conducted with $n=3$; mean \pm s.d. c Fluorescence images showing only one row of spots (cTnl) emitting bright fluorescence signals when a multiplexed antibody chip (two rows, two different antibodies against biomarkers labeled at the left of the image with different concentrations) were incubated in a solution containing a mixture of the two antigens, followed by incubation in a solution containing only one of the corresponding detection antibodies without the other one. $\mathbf{d}$ Averaged fluorescence intensity of each row in (c). All experiments were conducted with $n=3$; mean \pm s.d. Source data are provided as a Source Data file.

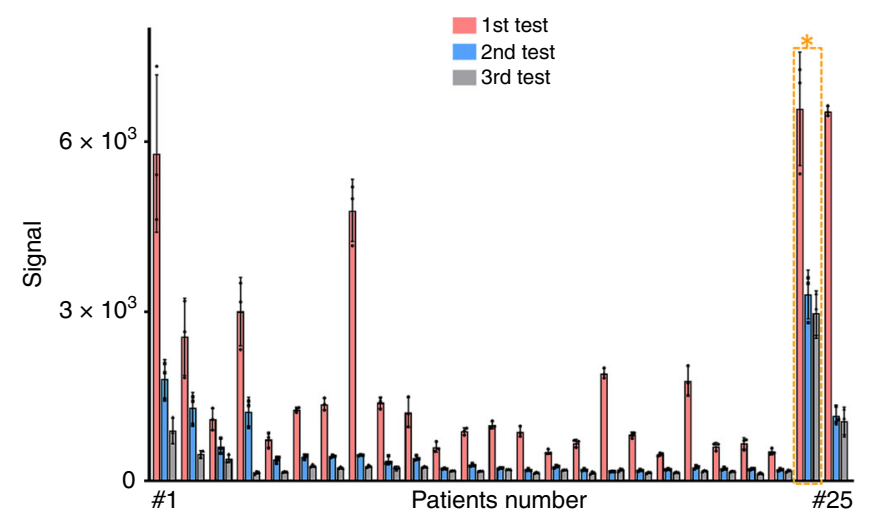

Fig. 5 Serum tests for monitoring after PCI using cTnl. Tri-tests of cTnl for all 25 patients in a period of 10 days, concluding 1st test results before $\mathrm{PCl}$ in red, 2nd test results on day 5 after $\mathrm{PCl}$ in black and 3rd test results on day 10 after $\mathrm{PCl}$ in blue. All experiments were conducted with $n=3$; mean \pm s.d. * referred to the MI patient (\#24, in the yellow box) that needed observation for a longer time after $\mathrm{PCl}$. Source data are provided as a Source Data file.

gradually decreased for the subsequent two tests after PCI for MI patients $(p<0.05)$, compared with levels of cTnI before PCI $(p<$ $0.0001)$. The gradual decrease of cTnI concentrations can be obtained in serum for all the 25 patients in three consecutive tests for short-term (10 days) outcome assessment that would be relevant to long-term (from 3 months to 8 years) morbidity and mortality for potential prognostic use $\mathrm{s}^{51-54}$.

PCI with around millions of cases per year (known as a preferred method of myocardial revascularization), is a commonly used technique for treating $\mathrm{MI}^{55}$. Notably, monitoring of PCI is crucial and decides the life quality for MI patients, while the cTnI level in serum serves as the main indicator in clinics. Considering the key role of cTnI and application benefit of our assay, the pGold chip assay may help to address the technical challenges and application needs dealing with MI from a clinical perspective, toward efficient detection and monitoring of MI in daily life.

To better put our findings into perspective, we included a small cohort of 10 patients (Supplementary Fig. 6 and Supplementary Table 9) presenting with acute chest pain to the emergency department of Shanghai Chest Hospital (intended use Population) and adjudicated the final diagnosis according to the universal definition using serial measurements of cTnI by pGold. In monitoring of cTnI, five patients (No. 1-5) with acute chest pain showed a significant elevation ( $\geq 20 \%$, according to the literature $)^{56,57}$ between two consecutive time points $(0 \mathrm{~h} / 1 \mathrm{~h}$ and $0 \mathrm{~h} / 3 \mathrm{~h})$, who were diagnosed with MI (in conjunction with clinical history, physical examination, and ECG) and required intervention treatment. For comparison, another five patients (No. 6-10) with acute chest pain showed no significant elevation between two consecutive time points $(0 \mathrm{~h} / 1 \mathrm{~h}$ and $0 \mathrm{~h} / 3 \mathrm{~h})$, who were diagnosed without MI (in conjunction with clinical history, physical examination, and ECG) and required no intervention treatment.

Our approach is different from previous reports owing to two major aspects, including disease diagnostics and application design. For disease diagnosis, we reported the assay based on plasmonic nanochips for diagnosis of myocardial infarction, distinct from previous reports using the similar plasmonic platform, such as diagnosis of type I child diabetes ${ }^{23}$ and Zika virus infection $^{26}$. For application design, this work contained the analytical features (compared with previous reports) ${ }^{58,59}$ as tailored, including multi-functions (monitoring for prognostic 
use) in clinics, selection of specific biomarkers (cTnI and CK-MB), and low sample consumption in high speed $(10 \mu \mathrm{L}$ of serum for $30 \mathrm{~min}$ reaction). In addition, our plasmonic gold nano-island platform afforded enhancement factors of $\sim 130$-fold, compared with the typical literatures ${ }^{58,59}$ with optimized enhancement factors of 50 -fold and 99-fold using plasmonic gold nanoparticles. Therefore, our approach addressed the application needs and technical challenges from a clinical perspective, different from typical literatures ${ }^{58,59}$ on sensing mechanism and device structure using plasmonic nanostructures to enhance the fluorescence of dyes from pure chemical/physical perspective.

In summary, we introduced pGold chips as a platform in the management of MI. Our approach featured for (1) quantitation of selected biomarkers (e.g., cTnI) at low concentrations down to $0.01 \mathrm{ng} \mathrm{mL}^{-1}$; (2) capability for detection of trace serum samples $(10 \mu \mathrm{L})$ toward POCT; (3) diagnosis of MI with the optimized sensitivity of $100 \%$ and specificity of $95.54 \%$; and (4) desirable speed and multiplexity for diagnostics and monitoring for prognostic use. This work contributed to the design of materials/device based POCT and the development of precision medicine for diverse diseases with tailored characteristics in near future.

\section{Methods}

Preparation of chips. Three types of chips were used, including glass chips, sputtered gold (sGold) chips, and plasmonic gold nano-island (pGold) chips. Glass chips with epoxy group were obtained from CapitalBio Company, China. pGold chips with gold nano-islands were obtained from Nirmidas Biotech Inc. and sGold chips were prepared by the magnetic sputtering method ${ }^{28}$. Glass slides were sputtered with gold using a magnetic control sputtering coater (GSL-1100X-SPC-16M) from Shenyang Kejing Auto-instrument Co., Ltd, China. Sputtering process was carried out at $40 \mathrm{~mA}$ current for $4 \mathrm{~min}$ under vacuum. Gold plate (99.99\%) for sputtering was ordered from Shanghai Daheng Optical Precision Machinery CO., LTD. Glass slides were immersed in chloroauric acid solution at the concentration of $3 \times 10^{-3} \mathrm{M}$. $\mathrm{NH}_{4} \mathrm{OH}$ was added by the volume ratio of 1:50 with rapid shaking for $1 \mathrm{~min}$ and immerged into deionized water for washing. Then slides were put into sodium borohydride solution at a concentration of $1 \times 10^{-3} \mathrm{M}$ for $1 \mathrm{~min}$ and washed with deionized water again. Finally, the slides were shifted to chloroauric acid solution and hydroxylamine solution at the mole ratio of 1:1 for $5 \mathrm{~min}$ of shaking and $10 \mathrm{~min}$ of incubation. Slides were dried after washing and stored in a sealable container.

Construction of device. Device was built in a manner for easy assembling. FAST frame slide holder and incubation chamber were ordered from Grace Bio-Labs, America. First, the chip was embedded into the incubation chamber and divided into 16 independent reaction wells. Then installed chip was loaded into FAST frame slide holder along two side grooves. The assembled device can be used to conduct immunoassays.

\section{Microarray printing. Nano-Plotter TM 2.1 (GeSim Co., Germany) was applied to immobilize the capture antibodies on the chips (including glass, sGold, and pGold chips). Chips with immobilized antibodies were prepared by the non-contact micro-dispensing method. Capture antibodies were diluted with $1 \times$ phosphate buffer saline (PBS, $\mathrm{pH}=7.4$ ) to the concentration of $3 \mu \mathrm{M}$. Then capture anti- bodies were printed in triplicate with each spot volume of $3 \mathrm{~nL}$ by running the designed printing program. The diameter of each spot was about $400 \mu \mathrm{m}$. The chip was placed at $4{ }^{\circ} \mathrm{C}$ before use.}

Characterization. For characterizations of chips, the extinction spectra of various chips were recorded with a UV1900 UV-Vis spectrometer (Aucybest, China). Scanning electron microscopy (SEM) images were acquired on Hitachi S-4800 with field emission gun (FEG) source at acceleration voltage of $10.0 \mathrm{kV}$. Digital images were recorded by iPhone 7 .

Antibody labeling by IRDye800. Detection antibodies were labeled with IRDye800 though 1-ethyl-3-(3-dimethyl aminopropyl) carbodiimide/N-hydroxysuccinimide (EDC-NHS) conjugation. Illustra NAP-5 columns were ordered from GE Healthcare and IRDye 800CW N-hydroxysuccinimide (NHS) ester was purchased from LI-COR Biosciences. IRDye 800CW NHS ester was diluted with dimethyl sulphoxide (DMSO) and detection antibody was mixed at the mole ratio of $1: 4$ and shaking for $1.5 \mathrm{~h}$ in the dark. First, $10 \mathrm{~mL}$ of $1 \times \mathrm{PBS}$ buffer $(\mathrm{pH}=7.4)$ was added into the column. Then the mixture was added with $1 \times$ PBS buffer $(\mathrm{pH}=7.4)$ to make the total volume of $500 \mu \mathrm{L}$ after dripping. Finally, IRDye800 labeled detection antibody solution was collected with $500 \mu \mathrm{L}$ of $1 \times$ PBS buffer $(\mathrm{pH}=7.4)$ and stored at $-20^{\circ} \mathrm{C}$ in dark before use.
Harvest of serum samples. All of the investigation protocols in this study were approved by the institutional ethics committees of the Shanghai Chest Hospital, Shanghai Jiao Tong University (Reference No. KS(P)1703). All serum samples detected in the experiment were collected in Shanghai Chest Hospital according to the established protocol ${ }^{60}$. Three milliliters of blood was drawn to a serum tube and centrifuged at $3000 \mathrm{rpm}$ for $15 \mathrm{~min}$. Aliquots of supernatant were collected and stored at $-20^{\circ} \mathrm{C}$ before use.

Immunoassay on chip. A sandwich structure was applied to detect serum biomarkers. Capture antibodies for cTnI (cat. \# 14T21, mAb: M18)/CK-MB (mAb: 1C11), Detection antibodies for cTnI (cat. \# 14T21, mAb: 19C7)/CK-MB (mAb: 1D10), and calibration materials for $\mathrm{cTnI} / \mathrm{CK}-\mathrm{MB}$ were obtained from Shanghai Tellgen Life Science Company. Printed chips (including glass, sGold, and pGold chips) were blocked with $5 \%$ bovine serum albumin (BSA) solution (diluted with $1 \times$ PBS buffer containing $0.05 \%$ tween-20) for $1 \mathrm{~h}$ shaking to reduce non-specific binding. Then, $150 \mu \mathrm{L}$ of $1 \times$ PBS buffer containing $0.05 \%$ tween-20 (PBST) was used to wash each reaction well. For calibration curves detection, calibration samples containing known concentrations of cTnI and CK-MB were diluted in pure water as the kit (Tellgen) suggested to a certain concentration. For serum samples detection, $10 \mu \mathrm{L}$ of human serum was diluted into $90 \mu \mathrm{L}$ of $10 \%$ fetal bovine serum (FBS) solution. Hundred microliters of solution from calibration sample and serum sample were added in each reaction well for 120 min of shaking at room temperature. After washing with PBST for five times, the chip was then stained IRDye800 labeled antibodies for anti-cTnI and anti-CK-MB at a concentration of $10 \times 10^{-9} \mathrm{M}$ for $30 \mathrm{~min}$ shaking in the dark. Obtained chips were washed three times by PBST. Hundred microliters of PBST and PBS were added into each reaction well for $15 \mathrm{~min}$ and $5 \mathrm{~min}$ shaking in the dark, subsequently. The chips were finally washed with deionized water and dried with compressed air before scanning.

NIR fluorescence measurement. The chips were scanned by InnoScan 710-IR microarray scanner using the $785 \mathrm{~nm}$ channel with a resolution of $10 \mu \mathrm{m}$ per pixel A 16-bit grayscale image can be exported after scanning. Then images were analyzed by Image Studio Version 3.1.4. Spot features were automatically identified by the program and then manually defined ${ }^{22}$. The intensity of each feature was calculated as the total signal divided by the area. The mean intensity of the three spots was used for quantitation.

Chemiluminescence immunoassay. Chemiluminescence immunoassay (CIA) was conducted on the UniCel DxI 800 (Beckman Coulter Inc.) using both $200 \mu \mathrm{L}$ (for the standard of care) and $10 \mu \mathrm{L}$ of serum. The CIA on the UniCel DxI 800 platform (Beckman Coulter) is a reported high-sensitivity cTnI assay in clinic use $\mathrm{e}^{61-63}$ and requires $200 \mu \mathrm{L}$ of serum for the optimal standard of care use to make the clinical diagnosis. Also, CIA based on one high-sensitivity cTnI assay (as recommended in the current guidelines ${ }^{37,40,46}$ ) was conducted using Abbott Architect kit (Abbott Architect STAT high-sensitivity cTnI reagent kit) and i2000SR platform (consuming $200 \mu \mathrm{L}$ of serum). For calibration curves, standard samples (containing known concentrations of cTnI/CK-MB) were re-dissolved by pure water. In serum analysis, $10 \mu \mathrm{L}$ of human serum was diluted into $90 \mu \mathrm{L}$ of $10 \%$ FBS solution (same as the gGold chip assay). Standard or serum samples were mixed with detection antibody for cTnI/CK-MB (conjugated with alkaline phosphatase) in a surfactantcontaining buffer. Then magnetic particles with capture antibodies were added. After incubation, magnetic particles were collected to measure the fluorescence based on the chemiluminescent substrate Lumi-Phos* 530 for quantitation.

LODs, LOQs, and cutoffs. LODs and LOQs were calculated for cTnI and CK-MB, according to the calibration curves by the pGold chips and CIA. The linear fitting equation of each calibration curve of two platforms was computed by OriginPro 8 . LOD was measured as the mean blank value plus 3 times of s.d. and LOQ was measured as the mean blank value plus 10 times of s.d. The intensity cutoffs were corresponding to serum concentrations of $0.04 \mathrm{ng} \mathrm{mL}^{-1}$ (recommended cutoff by $\mathrm{CIA}$ ) and $0.03 \mathrm{ng} \mathrm{mL}^{-1}$ (lowered cutoff by pGold) for cTnI, $4 \mathrm{ng} \mathrm{mL}^{-1}$ (recommended cutoff by CIA) and $3.13 \mathrm{ng} \mathrm{mL}^{-1}$ (lowered cutoff by pGold) for CK-MB

Diagnostic and monitoring experiments. Myocardial infarction (MI) was defined as pathologically as the death of myocardial cell due to the prolonged ischaemia ${ }^{57}$, which can be diagnosed on the basis of the 99th percentile of cTnI, clinical manifestation, ECG, and angiography according to the literature reports ${ }^{40,57}$. A case-control design was used and the 99th percentile of cTnI can be obtained using controls. The blood samples were obtained during an early screening of initial MI patients with chest pain and without treatment, who were later diagnosed with MI on the basis of the 99th percentile of cTnI, clinical manifestation, ECG, and angiography 40,57 . The cTnI concentration representing the 99 th percentile of the overall study population was $0.036 \mathrm{ng} \mathrm{mL}^{-1}\left(36 \mathrm{ng} \mathrm{L}^{-1}\right)$, consistent with the cutoffs of the current high-sensitivity cTnI assays $\left(14-52 \mathrm{ng} \mathrm{L}^{-1}\right)$. An elevation above the 99th percentile of cTnI is a mandatory criteria for the diagnosis of MI in vivo.

Typically, MI patients in China would first take blood tests for early screening purpose, then have coronary angiography for validation and potential percutaneous coronary intervention (PCI), according to the appropriate use criteria 
for coronary revascularization as reported ${ }^{64,65}$. For diagnostic application, MI serum samples were from $112 \mathrm{MI}$ patients $(75$ males and 37 females) with a median age of 64.39, who were diagnosed without malignant tumor, autoimmune disorders, severe infectious diseases, trauma, heart diseases, and other major diseases. Control serum samples were collected from 112 healthy controls $(81$ males and 31 females) with a median age of 61.66 , who were diagnosed without MI and other major diseases. Patients/controls known to have other medical conditions (such as active bleeding) were excluded. Both age and gender were matched with $p>0.05$ (according to Student's $t$-test and Fisher's exact test), between the MI patients and healthy controls.

For monitoring application, a cohort of 25 MI patients (19 males and 6 females) were analyzed with a median age of 68 (41-92). For past medical history, 5 patients had high blood pressure, 14 patients had no related diseases, and 6 patients had MI within recent 3 years. Three blood samples per patient were collected and monitored including one blood sample before PCI and two blood samples within 10 days (one on day 5 and the other on day 10) after PCI. Monitoring concentrations of $\mathrm{cTnI}$ after PCI can be critical in short-term (10 days in this work) outcome assessment that would be relevant to long-term (from 3 months to 8 years) morbidity and mortality according to literature reports ${ }^{51-54}$. Also, a small cohort of 10 patients presenting with acute chest pain to the emergency department of Shanghai Chest Hospital (intended use Population) was added and the final diagnosis was adjudicated according to the universal definition using serial measurements.

Control of reaction time. Reaction time of immunoassay was controlled to be 150 , 60 , and 30 min on pGold chips, including the capture and detection steps. Specifically, in the capture step, calibration samples containing known concentrations of cTnI $\left(0.08,0.02\right.$, and $\left.0.01 \mathrm{ng} \mathrm{mL}^{-1}\right)$ were incubated for 120,40 , and $20 \mathrm{~min}$, individually. In the detection step, antibody staining (by $2 \mathrm{nM}$ IRDye800 labeled detection antibodies) time was set to be 30,20 , and $10 \mathrm{~min}$, individually.

Cross reactivity tests. The procedure was the same with standards or serum detection on pGold chips except for the detection step. Calibration samples containing a mixture of $\mathrm{cTnI}\left(0.3-1.2 \mathrm{ng} \mathrm{mL}^{-1}\right)$ and CK-MB $\left(4.0-64.0 \mathrm{ng} \mathrm{mL}^{-1}\right)$ were applied to the pGold chips. In the detection step, one group of wells was added with only one IRDye800 labeled antibody, another group of wells added with the other antibody instead.

Statistical analysis. Coefficient variation was calculated as standard deviation divided by mean intensity. Positive cases among patient samples and negative cases among control samples were used to calculate the sensitivity (as true positive/(true positive + false negative)) and specificity (as true negative/(true negative + false positive)). Statistical Product and Service Solutions 19 (SPSS 19.0, SPSS Inc., Chicago) was used to plot and analyze the ROC curve. The CI and $p$-value were calculated using the SPSS software based on the Clopper-Pearson method, Fisher's exact test, unpaired Student's $t$-test, and Wilcoxon signed-rank test ${ }^{66-69}$.

Reporting summary. Further information on research design is available in the Nature Research Reporting Summary linked to this article.

\section{Data availability}

The datasets obtained and analyzed during the current study are available from the corresponding authors upon reasonable request. The source data for Figs. 1b, 2b, c, e, f, 3a, c, 4b, d, and 5, and Supplementary Figs. 2a, b, 3, 4, 5b, and 6 were provided as a Source Data file.

Received: 2 November 2018; Accepted: 13 March 2020;

Published online: 03 April 2020

\section{References}

1. Mendis, S., Puska, P. \& Norrving, B. Global Atlas on Cardiovascular Disease Prevention and Control (WHO, 2011).

2. Reiner, Z. Hypertriglyceridaemia and risk of coronary artery disease. Nat. Rev. Cardiol. 14, 401-411 (2017).

3. Benjamin, E. J. et al. Heart disease and stroke statistics-2017 update: a report from the American Heart Association. Circulation 135, e146-e603 (2017).

4. Prabhakaran, D. et al. Cardiovascular, respiratory, and related disorders: key messages from disease control priorities, 3rd edition. Lancet 391, 1224-1236 (2018).

5. Roth, G. A. et al. Global, regional, and national burden of cardiovascular diseases for 10 causes, 1990 to 2015. J. Am. Coll. Cardiol. 70, 1-25 (2017).

6. Sacks, F. M. et al. Dietary fats and cardiovascular disease: a presidential advisory from the American Heart Association. Circulation 136, e1-e23 (2017).
7. Braunwald, E. The war against heart failure: the Lancet lecture. Lancet $\mathbf{3 8 5}$, 812-824 (2015)

8. Jansen, F., Nickenig, G. \& Werner, N. Extracellular vesicles in cardiovascular disease: potential applications in diagnosis, prognosis, and epidemiology. Circ. Res. 120, 1649-1657 (2017).

9. Cohen, J. D. et al. Detection and localization of surgically resectable cancers with a multi-analyte blood test. Science 359, 926-930 (2018).

10. Nakamura, A. et al. High performance plasma amyloid-beta biomarkers for Alzheimer's disease. Nature 554, 249-254 (2018).

11. Wei, M. et al. Fasting-mimicking diet and markers/risk factors for aging, diabetes, cancer, and cardiovascular disease. Sci. Transl. Med. 9, 377-388 (2017).

12. Chaudhury, A., Noiret, L. \& Higgins, J. M. White blood cell population dynamics for risk stratification of acute coronary syndrome. Proc. Natl Acad. Sci. USA 114, 12344-12349 (2017).

13. Liu, C. et al. Quantification of circulating mycobacterium tuberculosis antigen peptides allows rapid diagnosis of active disease and treatment monitoring. Proc. Natl Acad. Sci. USA 114, 3969-3974 (2017).

14. Ngo, T. T. M. et al. Noninvasive blood tests for fetal development predict gestational age and preterm delivery. Science 360, 1133-1136 (2018).

15. Su, H. et al. Janus particles: design, preparation, and biomedical applications. Mater. Today Bio. 4, 100033 (2019).

16. Vedarethinam, V. et al. Detection and inhibition of bacteria on a dualfunctional silver platform. Small 15, 1803051 (2019).

17. Huang, L. et al. A multifunctional platinum nanoreactor for point-of-care metabolic analysis. Matter 1, 1669-1680 (2019).

18. Hong, G., Antaris, A. L. \& Dai, H. Near-infrared fluorophores for biomedical imaging. Nat. Bio. Eng. 1, 0010 (2017).

19. Shu, W. et al. Construction of a plasmonic chip for metabolic analysis in cervical cancer screening and evaluation. Small Methods. https://doi.org/ 10.1002/smtd.201900469 (2019).

20. Zhang, R. et al. Magnetic "squashing" of circulating tumor cells on plasmonic substrates for ultrasensitive NIR fluorescence detection. Small Methods 3, 1800474 (2019).

21. Tabakman, S. M. et al. Plasmonic substrates for multiplexed protein microarrays with femtomolar sensitivity and broad dynamic range. Nat. Commun. 2, 466 (2011).

22. Liu, B. et al. High performance, multiplexed lung cancer biomarker detection on a plasmonic gold chip. Adv. Funct. Mater. 26, 7994-8002 (2016).

23. Zhang, B., Kumar, R. B., Dai, H. \& Feldman, B. J. A plasmonic chip for biomarker discovery and diagnosis of type 1 diabetes. Nat. Med 20, 948-953 (2014).

24. Wan, H. et al. Proteoliposome-based full-length ZnT8 self-antigen for type 1 diabetes diagnosis on a plasmonic platform. Proc. Natl Acad. Sci. USA 114, 10196-10201 (2017).

25. Li, X. et al. Autoantibody profiling on a plasmonic nano-gold chip for the early detection of hypertensive heart disease. Proc. Natl Acad. Sci. USA 114, 7089-7094 (2017).

26. Zhang, B. et al. Diagnosis of Zika virus infection on a nanotechnology platform. Nat. Med. 23, 548-550 (2017).

27. Guidelli, E. J., Ramos, A. P. \& Baffa, O. Enhancing and quenching luminescence with gold nanoparticle films: the influence of substrate on the luminescent properties. Nanotechnology 27, 015503 (2016)

28. Sun, X. et al. Metabolic fingerprinting on a plasmonic gold chip for mass spectrometry based in vitro diagnostics. ACS Cent. Sci. 4, 223-229 (2018).

29. Caldarola, M. et al. Non-plasmonic nanoantennas for surface enhanced spectroscopies with ultra-low heat conversion. Nat. Commun. 6, 7915 (2015).

30. Liu, X., Zhou, J., Zhou, S., Yue, Y. \& Qiu, J. Transparent glass-ceramics functionalized by dispersed crystals. Prog. Mater. Sci. 97, 38-96 (2018).

31. Peng, X. et al. A nonfluorescent, broad-range quencher dye for Förster resonance energy transfer assays. Anal. Biochem. 388, 220-228 (2009).

32. Mayilo, S. et al. Long-range fluorescence quenching by gold nanoparticles in a sandwich immunoassay for cardiac troponin T. Nano Lett. 9, 4558-4563 (2009).

33. Xue, C., Xue, Y., Dai, L., Urbas, A. \& Li, Q. Size- and shape-dependent fluorescence quenching of gold nanoparticles on perylene dye. Adv. Opt. Mater. 1, 581-587 (2013).

34. Armbruster, D. A. \& Pry, T. Limit of blank, limit of detection and limit of quantitation. Clin. Biochem. Rev. 29, S49-S52 (2008).

35. Boeckel, J. N. et al. Estimation of values below the limit of detection of a contemporary sensitive troponin I assay improves diagnosis of acute myocardial infarction. Clin. Chem. 61, 1197-1206 (2015).

36. Lippi, G. et al. Analytical evaluation of the new Beckman Coulter Access high sensitivity cardiac troponin I immunoassay. Clin. Chem. Lab. Med. 56, 157-161 (2017)

37. Ungerer, J. P. J., Tate, J. R. \& Pretorius, C. J. Discordance with 3 cardiac troponin I and T assays: implications for the 99th percentile cutoff. Clin. Chem. 62, 1106-1114 (2016). 
38. Badertscher, P. et al. Effect of acute coronary syndrome probability on diagnostic and prognostic performance of high-sensitivity cardiac troponin. Clin. Chem. 64, 515-525 (2018).

39. Gimenez, M. R. et al. Impact of the US Food and Drug Administrationapproved sex-specific cutoff values for high-sensitivity cardiac troponin $\mathrm{T}$ to diagnose myocardial infarction. Circulation 137, 1867-1869 (2018).

40. Roffi, M. et al. 2015 ESC Guidelines for the management of acute coronary syndromes in patients presenting without persistent ST-segment elevation: Task force for the management of acute coronary syndromes in patients presenting without persistent ST-segment elevation of the European Society of Cardiology (ESC). Eur. Heart J. 37, 267-315 (2016).

41. Keller, T. et al. Sensitive troponin I assay in early diagnosis of acute myocardial infarction. N. Engl. J. Med. 361, 868-877 (2009).

42. Keller, T. et al. Defining a reference population to determine the 99th percentile of a contemporary sensitive cardiac troponin I assay. Int. J. Cardiol. 167, 1423-1429 (2013)

43. Shah, A. S. V. et al. High-sensitivity troponin in the evaluation of patients with suspected acute coronary syndrome: a stepped-wedge, cluster-randomised controlled trial. Lancet 392, 919-928 (2018).

44. Reichlin, T. et al. Early diagnosis of myocardial infarction with sensitive cardiac troponin assays. N. Engl. J. Med. 361, 858-867 (2009).

45. Giménez, M. R. et al. Rapid rule out of acute myocardial infarction using undetectable levels of high-sensitivity cardiac troponin. Int. J. Cardiol. 168, 3896-3901 (2013).

46. Love, S. A. et al. Incidence of undetectable, measurable, and increased cardiac troponin I concentrations above the 99th percentile using a high-sensitivity vs a contemporary assay in patients presenting to the emergency department. Clin. Chem. 62, 1115-1119 (2016).

47. Chiara, A. D. et al. Predictive value of cardiac troponin-I compared to creatine kinase-myocardial band for the assessment of infarct size as measured by cardiac magnetic resonance. J. Cardiovasc. Med. 11, 587-592 (2010).

48. Søraas, C. L. et al. Troponin T is a better predictor than creatine kinase-MB of long-term mortality after coronary artery bypass graft surgery. Am. Heart J. 164, 779-785 (2012).

49. Neumann, J. T. et al. Diagnosis of myocardial infarction using a highsensitivity troponin I 1-hour algorithm. JAMA Cardio 1, 397-404 (2016).

50. Boeddinghaus, J. et al. Two-hour algorithm for triage toward rule-out and rule-in of acute myocardial infarction by use of high-sensitivity cardiac troponin I. Clin. Chem. 62, 494-504 (2016).

51. Kizer, J. R. et al. Role of cardiac troponin $\mathrm{T}$ in the long-term risk stratification of patients undergoing percutaneous coronary intervention. Eur. Heart J. 24, 1314-1322 (2003).

52. Tricoci, P. et al. Cardiac troponin after percutaneous coronary intervention and 1-year mortality in non-ST-segment elevation acute coronary syndrome using systematic evaluation of biomarker trends. J. Am. Coll. Cardiol. 62, 242-251 (2013).

53. Hall, T. S. et al. Cardiac troponin I for prediction of clinical outcomes and cardiac function through 3-month follow-up after primary percutaneous coronary intervention for ST-segment elevation myocardial infarction. Am. Heart J. 169, 257-265 (2015).

54. Ndrepepa, G. et al. Markers of reperfusion and long-term (8-year) prognosis after primary percutaneous coronary intervention. Am. J. Cardiol. 122, 39-46 (2018).

55. Shah, R. U. et al. Increasing percutaneous coronary interventions for STsegment elevation myocardial infarction in the United States: progress and opportunity. JACC Cardiovasc. Interv. 8, 139-146 (2015).

56. Wang, Y. et al. Chinese experts' consensus on the clinical application of cTnI. Chin. J. Inter. Med. 54, 899-904 (2015).

57. Thygesen, K. et al. Fourth universal definition of myocardial infarction. J. Am. Coll. Cardiol. 72, 2231-2264 (2018).

58. Chekini, M. et al. Fluorescence enhancement in large-scale self-assembled gold nanoparticle double arrays. J. Appl. Phys. 118, 233107 (2015).

59. Bardhan, R., Grady, N. K. \& Halas, N. J. Nanoscale control of near-infrared fluorescence enhancement using Au nanoshells. Small 4, 1716-1722 (2008).

60. Wu, J. et al. Multifunctional magnetic particles for combined circulating tumor cells isolation and cellular metabolism detection. Adv. Funct. Mater. 26, 4016-4025 (2016).

61. Kavsak, P. A., Malinowski, P., Roy, C., Clark, L. \& Lamers, S. Assessing matrix, interferences and comparability between the Abbott Diagnostics and the Beckman Coulter high-sensitivity cardiac troponin I assays. Clin. Chem. Lab. Med 56, 1176-1181 (2018).

62. Masotti, S. et al. Evaluation of analytical performance of a new high-sensitivity immunoassay for cardiac troponin I. Clin. Chem. Lab. Med. 56, 492-501 (2018).
63. Clericoa, A. et al. Evaluation of 99th percentile and reference change values of a high-sensitivity cTnI method: a multicenter study. Clin. Chim. Acta 493, 156-161 (2019).

64. Patel, M. R. et al. ACC/AATS/AHA/ASE/ASNC/SCAI/SCCT/STS 2017 appropriate use criteria for coronary revascularization in patients with stable ischemic heart disease. J. Am. Coll. Cardiol. 69, 2212-2241 (2017).

65. Desai, N. R. et al. Appropriate use criteria for coronary revascularization and trends in utilization,patient selection, and appropriateness of percutaneous coronary intervention. JAMA 314, 2045-2053 (2015).

66. Norton, M. E. et al. Cell-free DNA analysis for noninvasive examination of trisomy. N. Engl. J. Med. 372, 1589-1597 (2015).

67. Evans, C. P. et al. The PREVAIL study: primary outcomes by site and extent of baseline disease for enzalutamide-treated men with chemotherapynaive metastatic castration-resistant prostate cancer. Eur. Urol. 70, 675-683 (2016).

68. Huang, L. et al. Plasmonic silver nanoshells for drug and metabolite detection. Nat. Commun. 8, 220 (2017).

69. Meldi, K. et al. Specific molecular signatures predict decitabine response in chronic myelomonocytic leukemia. J. Clin. Invest. 125, 1857-1872 (2015).

\section{Acknowledgements}

We gratefully thank the financial support from Project 81771983, 81971771, $81750410695,81750110544,81650110523,81851110764$, and 61425006 by National Natural Science Foundation of China (NSFC), Project 16441909300 by Shanghai Science and Technology Commission, Project YS2017YFGH002082, 2017YFC0909000 by Ministry of Science and Technology of China. This work is also sponsored by Shanghai Rising-Star Program (19QA1404800), Clinical Research Plan of SHDC (16CR2011A), and Innovation Group Project of Shanghai Municipal Health Commission (2019CXJQ03).

\section{Author contributions}

K.Q. and J.L. foresaw this work and designed the overall approach. W.X. and L.W. contributed equally. W.X., L.W., and R.Z. carried out experiments and wrote the paper. X.S., L.H., H.S., C.C., X.W., J.L., and H.D. contributed to the data analysis. All authors joined in the critical discussion and edited the paper.

\section{Competing interests}

The authors declare the following competing interests. H.D. is a scientific adviser for Nirmidas Biotech Inc. and contributed to this work in that capacity. The authors declare no other competing interests.

\section{Additional information}

Supplementary information is available for this paper at https://doi.org/10.1038/s41467 020-15487-3.

Correspondence and requests for materials should be addressed to J.L. or K.Q.

Peer review information Nature Communications thanks Christian Mueller and the other, anonymous, reviewer(s) for their contribution to the peer review of this work

Reprints and permission information is available at http://www.nature.com/reprints

Publisher's note Springer Nature remains neutral with regard to jurisdictional claims in published maps and institutional affiliations.

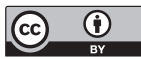

Open Access This article is licensed under a Creative Commons Attribution 4.0 International License, which permits use, sharing adaptation, distribution and reproduction in any medium or format, as long as you give appropriate credit to the original author(s) and the source, provide a link to the Creative Commons license, and indicate if changes were made. The images or other third party material in this article are included in the article's Creative Commons license, unless indicated otherwise in a credit line to the material. If material is not included in the article's Creative Commons license and your intended use is not permitted by statutory regulation or exceeds the permitted use, you will need to obtain permission directly from the copyright holder. To view a copy of this license, visit http://creativecommons.org/ licenses/by/4.0/.

(C) The Author(s) 2020 